\title{
$\begin{array}{ll}\text { Research Square } & \text { Preprints are preliminary reports that have not undergone peer review. } \\ \text { They should not be considered conclusive, used to inform clinical practice, } & \text { or referenced by the media as validated information. }\end{array}$
}

\section{Molecular subtyping and prognostic assessment of prostate cancer based on consensus genes}

\section{Jialin Meng}

Department of Urology, The First Affiliated Hospital of Anhui Medical University

\section{Yu Guan}

Department of Urology, The First Affiliated Hospital of Anhui Medical University

\section{Bijun Wang}

Department of Urology, The First Affiliated Hospital of Anhui Medical University

\section{Lei Chen}

Department of Urology, The First Affiliated Hospital of Anhui Medical University

\section{Junyi Chen}

Department of Urology, The First Affiliated Hospital of Anhui Medical University

\section{Meng Zhang}

Department of Urology, The First Affiliated Hospital of Anhui Medical University Chaozhao Liang ( $\square$ liang_chaozhao@ahmu.edu.cn )

Department of Urology, The First Affiliated Hospital of Anhui Medical University

\section{Article}

Keywords: Prostate cancer, molecular subtyping, consensus gene, classifier, recurrence-free survival

Posted Date: April 21st, 2021

DOI: https://doi.org/10.21203/rs.3.rs-413181/v1

License: (c) (i) This work is licensed under a Creative Commons Attribution 4.0 International License. Read Full License

Version of Record: A version of this preprint was published at Communications Biology on March 15th, 2022. See the published version at https://doi.org/10.1038/s42003-022-03164-8. 


\section{Abstract}

Prostate cancer ( $\mathrm{PCa}$ ) is one of the most common malignancies in men all over the world. We performed molecular subtyping and prognostic assessment based on consensus genes in patients with PCa. Five cohorts containing 1,046 PCa patients with RNA expression profiles and recorded clinical follow-up information were included. Univariate, multivariate Cox regression analysis and the Least Absolute Shrinkage and Selection Operator (LASSO) Cox regression was used to select prognostic genes and establish the signature. Immunohistochemistry staining, cell proliferation, migration and invasion assay were used to assess the biological function of key genes. 39 intersecting consensus prognostic genes from five independent cohorts were identified $(P<0.05)$. Subsequently, an eleven-consensus-gene-based classifier was established. Besides, multivariate Cox regression analyses proved that the classifier served as an independent indicator of recurrence-free survival in three of five cohorts. Combined receiver operating character (ROC) achieved synthesized effects by combining the classifier with clinicopathological features in four of five cohorts. SRD5A2 inhibits the cell proliferation, while ITGA11 promote cell migration and invasion, of which might through PI3K/AKT signaling pathway. To conclude, we establish and validate an eleven-consensus-gene-based classifier, which adds prognostic value to the currently available staging system.

\section{1 | Introduction}

Prostate cancer ( $\mathrm{PCa}$ ) is one of the most common malignancies in men all over the world ${ }^{1,2}$. Although the majority of localized PCa patients can be cured by surgery and/or radiation therapy, part of the PCa patients still face the severe situation of progressing to castration-resistant PCa (CRPC) ${ }^{3,4}$. On the contrary, some patients have indolent tumors, which rarely progress to an advanced stage or influence their life-quality. Thus, it is crucial to identify patients with the high-risk of recurrence before therapy.

Gleason score is a widely used feature to reflect the degree of malignancy for $\mathrm{PCa}{ }^{5-8}$. Along with the published studies, patients with Gleason score $<=6$ rarely suffer threaten of death, while those patients with Gleason score $>8$ frequently confront the fact of tumor progression ${ }^{9}$. Although histological examination easily discriminates the tumors with Gleason score $<6$ or $>8$, it is still hard to verify the patients with an intermediate stage (Gleason score $3+4$ or $4+3)^{10}$. Commonly, PCa patients with Gleason score of $3+4$ are less aggressive than those with $4+3$. However, sampling error, bias among different pathologists, the subjectivity of assessing are the obvious confounding factors for the misleading results ${ }^{11}$. Therefore, it is urgent to develop a universal molecular classifier to recognize patients with high-risk of poor prognosis.

Since the early 2000s, gene expression profiles have been applied to classify early-stage tumor patients into different subtypes based on the molecular markers, and these subtypes of patients were correlated with different outcomes or clinicopathological features. Further, studies also indicated that the gene expression panel test could guide in tailoring treatment decisions for doctors, promoting the development of personalized medicine ${ }^{12}$. Recently, increasing evidence showed that gene expression-based classifiers 
may be useful for disease classification independent of the available prognostic factors, severing as the clinical implementation. Although the gene expression classifier could be constructed and validated in the publicly released dataset, or even a single-center study, there remains a gap invalidating the identified classifier in large cohorts.

We aimed to assess the usage of consensus recurrence-free survival (RFS) associated genes derived from five independent cohorts to generate the molecular subtyping with different clinical outcome for PCa patients.

\section{2 | Materials And Methods}

\section{1 | Data preparation and processing}

We searched the Gene Expression Omnibus (GEO: http://www.ncbi.nlm.nih.gov/geo/) to enroll the eligible datasets which meet the following criteria: 1) PCa cases with available expression data; and 2) with available clinicopathological features, particularly for RFS status and time. Then, the gene expression profiles were generated from four eligible GEO datasets [GSE116918, GSE70769, GSE70768, and GSE21032/Memorial Sloan Kettering Cancer Center (MSKCC)], as well as the gene expression profile from The Cancer Genome Atlas (TCGA) database (https://cancergenome.nih.gov). The matched clinicopathological data were also downloaded along with the expression profiles. Patients who lack the data of the pathology $T$ stage were excluded.

\section{2 | Univariate Cox regression analysis and consensus gene selection}

Univariate Cox regression analysis was conducted to screen out the consentaneous RFS-related genes observed from the five datasets. Subsequently, we intersected these RFS-related candidates that appeared in all five datasets, with a cut-off $P$-value of less than 0.05 . The following analyses were performed based on these selected genes.

\section{3 | Classifier establishment and validation}

According to the results provided by univariate Cox regression analysis, we employed LASSO Cox regression to select more stable candidates. The classifier was established referring to the expression and co-efficient of each candidate based on the MSKCC cohort. We calculated the risk score for each patient along with the below formula:

$$
\sum_{i=1}^{n}[\operatorname{coef}(\operatorname{mRNA} i) * \operatorname{Expression}(\mathrm{mRNA} i)]
$$

Median value of the risk score set as the cut-off value in each cohort, and for these patients with lower risk scores than the median value were assigned into the low-risk subgroup, while others belonged to the high-risk subgroup. The risk score of patients in the other four external validation cohorts was also 
calculated by this risk formula and then dichotomized these patients into two different risk subgroups by the median risk score in each cohort.

\section{4 | Survival and receiver operating characteristic (ROC) analyses}

Survival analyses were executed using the "survminer" package, with the RFS as the endpoint. Furthermore, the area under the ROC curve (AUC) was employed to assess the predictive value of the formula. Furthermore, subgroup analyses were executed to test the accuracy of the classifier in different clinicopathological subgroups, such as different Gleason score ( $<=7 \mathrm{vs} .>7)$, pathological tumor stage $(\mathrm{T} 1+\mathrm{T} 2$ vs. $\mathrm{T} 3+\mathrm{T} 4)$, and age (<= 60 vs. $>60)$.

\section{5 | Immunohistochemistry (IHC) validation}

To validate the association between SRD5A2 and ITGA11 and the clinicopathological features, we used the IHC assay to detect the protein expression of the above two genes in prostate cancer tissue array (Outdo Biotech Co., Ltd., Shanghai, China), which contains the tumor tissue from 42 patients. The detailed steps of the IHC procedure have been previously reported ${ }^{13,14}$. The antibodies of SRD5A2 (Cat. \#: DF8416, Affinity Biosciences LTD., Ohio, USA) and ITGA11 (Cat. \#: bs-13771R, Bioss Antibodies LTD., Massachusetts, USA) were applied for the IHC staining with a dilution of 1:250. We recorded the staining intensity as: 0 , negative; 1 , weak positive; 2 , moderate positive; and 3 , strong positive. Besides, the staining area was indicated as: $0,0 \% ; 1,1-25 \% ; 2,26-50 \% ; 3,51-75 \%$; and $4,>76 \%$. The intensity score multiplied with staining area were defined as the ultimate scores $(>=3$, positive staining; $<3$, negative staining) ${ }^{15}$.

\section{6 | Cell culture and knockdown of SRD5A2 and ITGA11}

We cultured the C4-2 and PC-3 cell lines with RPMI 1640 medium, which also added 10\% fetal bovine serum and $1 \%$ penicillin and streptomycin, cells cultured in the condition of $37^{\circ} \mathrm{C}$ and $5 \% \mathrm{CO}_{2}$. We obtained the $1 \times 10^{8} \mathrm{TU} / \mathrm{ml}$ shSRD5A2 and shITGA11 lentivirus from Shanghai Novobio Co, Ltd (Shanghai, China). To obtain the lentivirus, the shITGA11-1\#, shITGA11-2\#, shSRD5A2-1\#, shSRD5A2-2\# were inserted into the PDS126_pL-U6-shRNA-GFP vector. The knockdown sequences showed as follow: sh/TGA11-1\#-F: GCTCTTACTTTGGGAGTGAAA, sh/TGA11-1\#-R: TTTCACTCCCAAAGTAAGAGC; shITGA11-2\#-F: GCCATCCAAGATCAACATCTT, shITGA11-2\#-R: AAGATGTTGATCTTGGATGGC; shSRD5A21\#-F: GTGGTGTCTGCTTAGAGTTTA, shSRD5A2-1\#-R: TAAACTCTAAGCAGACACCAC; shSRD5A2-2\#-F: CTCAATCGAGGGAGGCCTTAT, shSRD5A2-2\#-R: ATAAGGCCTCCCTCGATTGAG.

\section{7 | Assay of cell proliferation, migration, and invasion}

To evaluate the impact of SRD5A2 and ITGA11 on prostate cancer cells, we employed MTT assay and colony formation assay to assess the alteration of cell proliferation, while Transwell-based invasion and migration assay was used to evaluate the ability of cell migration and invasion. 
For MTT assay, 5000 cells seeded in per well of 24-well plates, and then collected the results by add 50 $\mu \mathrm{L}$ prepped $5 \mathrm{mg} / \mathrm{mL}$ MTT reagent to $450 \mu \mathrm{L}$ refreshed medium (with a concentration of $0.5 \mathrm{mg} / \mathrm{mL}$ ) and incubated at $37^{\circ} \mathrm{C}$ for 1.5 hours. On the sixth day, add the DMSO solution to all plates $(0,2$ days, 4 days, and 6 days) to dissolve the formazan crystals and then read the optical density value at $570 \mathrm{~nm}$ to display the cell viability. For colony formation, 800 cells were seeded per well and grew for 12 days. The cells in plates will be fixed by $4 \%$ paraformaldehyde for 20 minutes and then the $0.05 \%$ crystal violet was used to stain these fixed cells for another 20 minutes.

For migration assay, Transwell Permeable Supports (Corning Inc., Maine, USA) was used. A total of $1 \times$ $10^{5}$ cells was seeded in the upper chamber for each well, and $500 \mu \mathrm{L}$ fresh medium with $10 \%$ FBS added into the lower chamber, and then incubated at $37^{\circ} \mathrm{C}$ and $5 \% \mathrm{CO}_{2}$ incubator for 24 hours. The cells migrated to the bottom of the membranes were permeabilized by methanol and stained with $0.01 \%$ Crystal Violet. The steps of invasion assay are similar to migration assay, which also used Permeable Supports, but with extra Matrigel (Biocoat, Corning, New York, USA) diluted and coated at the upper chambers and incubated for 36 hours. The cell numbers were calculated by counting three random fields.

\section{8 | Functional prediction}

Increasing evidence indicated that highly co-expressed genes potentially have similar biological functions 16-18, and we used Pearson correlation analysis to find out the highly co-expressed genes of ITGA11 on the five cohorts, respectively. After overlapping these co-expressed genes, we performed the Kyoto Encyclopedia of Genes and Genomes (KEGG) pathway enrichment analysis to subclassify their functions based on the "clusterProfiler" R package ${ }^{19}$. Besides, we also used Cytoscape (v3.5.1, San Diego, La Jolla, California, USA) to visualize the functional network. Two external gene sets, HALLMARK PI3K/AKT SIGNALING and REACTOME PI3K/AKT ACTIVATION, were employed to assess the association between ITGA11 expression and the activation PI3K signaling pathway. The single sample gene set enrichment analysis (ssGSEA) ${ }^{20,21}$, implemented in the GSVA R package, was applied to calculate the normalized enrichment score (NES) of the above 2 gene sets.

\section{9 | Western blot validation}

Proteins $(40-50 \mu \mathrm{g})$ were separated on $12.5 \%$ SDS/PAGE gels then transferred onto nitrocellulose blotting membranes (GE Healthcare Life Science, Germany). Membranes were blocked by $5 \%$ Bovine Serum Albumin (Sigma-Aldrich, St. Louis, MO, USA) for 1 hour at room temperature and then incubated with appropriate dilutions of specific primary antibodies against SRD5A2 (Cat. \#: DF8416, Affinity Biosciences LTD., Ohio, USA), ITGA11 (Cat. \#: bs-13771R, Bioss Antibodies LTD., Massachusetts, USA), AKT1/2/3 (Cat. \#: AF6216, Affinity Biosciences LTD., Ohio, USA), p-AKT1/2/3 (Ser ${ }^{473}$ ) (Cat. \#: AF0016, Affinity Biosciences LTD., Ohio, USA), GAPDH (Cat. \#: 1049-1-AP, Proteintech Group, Illinois, USA) overnight at $4^{\circ} \mathrm{C}$. Next day, after incubating with HRP-conjugated secondary antibodies for one hour, the membranes were visualized using the ECL system (Pierce; Thermo Fisher Scientific, Inc., USA). 


\subsection{0 | Statistics}

All the statis Comparisons of continuous data between two subtypes were performed by Student's t-test and Mann-Whitney $U$ test for normal and non-normal distribution data. Correlations between staining intensity subgroups and clinicopathological subgroups were evaluated by Fisher's exact test. Spearman's correlation analysis was utilized to explore the correlation between continuous variables. For all statistical analyses, a two-tailed $P$ value less than 0.05 was considered statistically significant.

\section{3 | Results}

\section{1 | Construction of the eleven-consensus-gene-based classifier}

In the current study, we enrolled a total of 1,046 PCa patients from the MSKCC $(n=140)$, TCGA-PRAD $(n=$ 489), GSE116918 $(n=223), \operatorname{GSE70769}(n=85)$, GSE70768 $(n=109)$ datasets. The clinicopathological features of all the PCa patients were listed in Table 1. We overlapped the significant genes $(P<0.05)$ generated by univariate Cox regression analyses in five independent cohorts, and found that there were 39 consensus candidates, and significantly associated with the RFS of PCa patients in all the five datasets (Fig. 1A, and Table S1). The expression landscape of these 39 genes in the MSKCC cohort showed in Fig. 1B. To obtain a more stable and significant consensus-gene-cluster-based classifier, we employed the LASSO Cox analysis based on the MSKCC dataset. Finally, eleven RFS-related consensus genes were selected, including MYBPC1, DPP4, UBE2J1, KIF13B, SRD5A2, OGN, NOX4, ITGA11, COL 1A1, STMN1, and CDKN3 (Fig. 1C-D). Then, the risk score of each patient was calculated by the prognostic model: 0.541407161 * $C D K N 3+0.986301077$ * COL $1 A 1+0.055793216$ * DPP4+ 1.204285151 * ITGA11-0.329370122 * KIF13B -0.353092775 * MYBPC1+0.228495902 * NOX4-0.374366498 * OGN $-0.711376668 *$ SRD5A2+0.492742742* STMN1 +0.417671671 * UBE2J1. 
Table 1

Summary of the clinicopathological parameters of five independent prostate cancer datasets.

\begin{tabular}{|c|c|c|c|c|c|}
\hline Items & $\begin{array}{l}\text { MSKCC } \\
(n=140)\end{array}$ & $\begin{array}{l}\text { TCGA-PRAD } \\
(n=489)\end{array}$ & $\begin{array}{l}\text { GSE70768 } \\
(n=109)\end{array}$ & $\begin{array}{l}\text { GSE70769 } \\
(n=85)\end{array}$ & $\begin{array}{l}\text { GSE116918 } \\
(n=223)\end{array}$ \\
\hline \multicolumn{6}{|l|}{ Age } \\
\hline 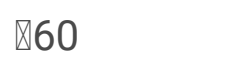 & 87 & 219 & 42 & - & 26 \\
\hline$\geq 60$ & 53 & 270 & 67 & - & 197 \\
\hline \multicolumn{6}{|c|}{ Pathology T grade } \\
\hline $\mathrm{T} 1+\mathrm{T} 2$ & 86 & 187 & 34 & 46 & 127 \\
\hline $\mathrm{T} 3+\mathrm{T} 4$ & 54 & 302 & 75 & 39 & 96 \\
\hline \multicolumn{6}{|l|}{ Gleason\# } \\
\hline 5 & - & - & - & 2 & - \\
\hline 6 & 41 & 44 & 18 & 16 & 39 \\
\hline 7 & 76 & 243 & 82 & 54 & 88 \\
\hline 8 & 11 & 61 & 8 & 5 & 47 \\
\hline 9 & 10 & 138 & 1 & 7 & 49 \\
\hline 10 & - & 3 & - & 1 & - \\
\hline \multicolumn{6}{|l|}{$\mathrm{PSA}^{*}$} \\
\hline$>10 \mathrm{ng} / \mathrm{ml}$ & 24 & 15 & 26 & 26 & - \\
\hline$\leq 10 \mathrm{ng} / \mathrm{ml}$ & 114 & 417 & 82 & 59 & - \\
\hline \multicolumn{6}{|c|}{ \#Gleason score information is missing in 2 patients from MSKCC cohort } \\
\hline
\end{tabular}

The median risk score was set as the cut-off value in each cohort, and these patients with lower risk scores were assigned into the low-risk subgroup, while others were classified into the high-risk subgroup (Table S2). Further, we correlated the expression of the eleven genes with clinicopathological features, and the results indicated that UBE2J1, SRD5A2, OGN, MYBPC1, KIF13B, and DPP4 were negatively correlated with Gleason score, PSA level, and pathological tumor stage, while opposite results were obtained for STMN1, NOX4, ITGA11, COL1A1, and CDKN3 genes (Fig. 1E).

\section{2 | Analysis results based on consensus genes and prognostic assessment in five cohorts}


Referring to the subgroups, in the training MSKCC cohort, 70 patients were divided into the high-risk group, and other 70 patients belonged to the low-risk group (Fig. 2A), and patients in the high-risk subgroup showed an unfavorable prognosis (log-rank $P<0.001$, Fig. 2F), with the AUC values of 0.908 in 1-year, 0.898 in 3-year, and 0.857 in 5-year (Fig. 2K). The predicting classifier also applied well in four external datasets. The K-M curves showed the similar RFS outcomes in GSE116918 (log-rank, $P<0.001$ ), GSE70768 (log-rank, $P=0.049$ ), GSE70769 (log-rank, $P<0.001$ ) and TCGA-PRAD cohorts (log-rank, $P<$ 0.001) (Fig. 2B-E, G-J). The classifier showed a moderate predictive accuracy in all the four validation cohorts (AUC values of $0.936,0.735$, and 0.705 at $1-, 3-, 5$-year in the GSE1 16918 cohort; AUC values of $0.816,0.706$, and 0.554 at $1-, 3-, 5$-year in the GSE70768 cohort; AUC values of $0.858,0.806$, and 0.745 at 1 -, 3-, 5-year in the GSE70769 cohort; AUC values of $0.717,0.711$, and 0.641 at $1-, 3-, 5$-year in the TCGAPRAD cohort, Fig. 2L-0).

To further investigate the clinical application value of the eleven-consensus-gene-based classifier in different clinicopathological subgroups, K-M analyses were performed. The signature precisely subclassified the high- and low-risk groups of PCa patients into different subgroups with adequate samples but failed in some conditions, potentially attributing to the small sample size (Fig. 3, Figure S1). For example, in the MSKCC cohort, which comprised 140 PCa cases, and the results suggested that the classifier significantly discriminated the high- and low-risk subgroups in different age ( $>=60$ vs. $<60$ years old), PSA level (> $10 \mathrm{vs} .<=10 \mathrm{ng} / \mathrm{dl})$, pathological tumor stage (T3+T4 vs. T1 + T2), and Gleason score (> 7 vs. $<=7$ ) subgroups (All, log-rank $P<0.05$ ). For the GSE70769 cohort, which comprised $85 \mathrm{PCa}$ cases, we also revealed the classifier significantly discriminated the high- and low-risk subgroups of the different PSA (> 10 vs. $<=10$ ), tumor stage (T3 + T4 vs. T1 + T2) subgroups (log-rank $P<0.05$ ), Gleason score (>7 vs. $<=7$ ) subgroups, and surgical margin (negative vs. positive) (All, log-rank $P<0.05$ ). Further well-designed studies are warranted to verify our findings.

Notably, the clinical outcomes of PCa patients with Gleason score $3+4$ and $4+3$ are different. It is necessary to distinguish these two subgroups with not only pathological results. We investigated the value of this classifier in distinguishing patients with Gleason score $3+4$ and $4+3$ subgroups in MSKCC, TCGA-PRAD, GSE70768, and GSE70769 datasets, while the GSE116918 missed this feature and was removed from the analysis. We observed that patients with Gleason score $4+3$ have a higher risk score as compare with Gleason score $3+4$ patients (MSKCC, $P=0.092$; TCGA-PRAD, $P<0.001$; GSE70768, $P=$ 0.006; GSE70769, $P=0.007$ ). Besides, the risk score distinguished the $3+4 / 4+3$ subgroups with good accuracy (MSKCC, AUC = 0.636; TCGA-PRAD, AUC = 0.718; GSE70768, AUC = 0.712; GSE70769, AUC = 0.731). As confirmed by Fisher's Extract test, we found that more patients with the high-risk belonged to the Gleason score $4+3$ subgroup (MSKCC, $P=0.079$; TCGA-PRAD, $P<0.001$; GSE70768, $P=0.024$; GSE70769, $P=0.010$ ) (Figure S2).

\section{3 | Analysis results of multivariate Cox regression, and Combined ROC}


To determine the independence of the eleven-consensus-gene-based classifier in each cohort, we performed multivariate Cox regression analyses. Our results showed that for the cohorts, whose recurrence rate $>15 \%$, the classifier was serving as an independent indicator for RFS (MSKCC cohort: HR $=5.44,95 \%$ Cl: $1.83-16.16, P=0.002 ;$ GSE116918 cohort: HR = 3.64, 95\% Cl: $1.82-7.29, P<0.001$; GSE70769 cohort: HR $=2.53,95 \%$ Cl: $1.22-5.24, P=0.013$; TCGA-PRAD cohort: HR $=1.39,95 \%$ Cl: $0.76-$ 2.52, $P=0.282$; GSE70768 cohort: HR $=0.818,95 \% \mathrm{Cl}: 0.29-2.28, P=0.701$; Fig. 4A-C, and Figure S3A-B).

In addition, we performed Combined ROC analyses by combining the classifier with critical clinicopathological features in each cohort. We found that the Combined obtained an increased predictive values than each variable alone in the MSKCC (AUC $=0.89,95 \% \mathrm{Cl}: 0.81-0.97), \mathrm{GSE} 116918$ (AUC $=0.69$, $95 \%$ Cl: $0.59-0.79$ ), GSE70769 (AUC $=0.82,95 \%$ Cl: 0.73-0.91), TCGA-PRAD (AUC $=0.75,95 \%$ Cl: $0.68-$ 0.83), GSE70768 (AUC $=0.76,95 \% \mathrm{Cl}$ : 0.60-0.92) cohorts (Fig. 4D-F, and Figure S3C-D).

\section{4 | IHC validation the protein of SRD5A2 and ITGA11}

To address and confirm the associations of SRD5A2 and ITGA11 protein levels with clinicopathological features, we used IHC assay on prostate cancer tissue array, which contains tumor tissues from 42 patients. The standard definition of the SRD5A2 protein level was mentioned in the Methods section. Then, we calculated the staining density of each tissue. Tissues with score equal to or higher than 3 were regarded as positive, while those with score less than 3 were negative.

We observed the decreased protein expression of SRD5A2 in advanced tumors stage (Gleason $<=7 \mathrm{vs}$. Gleason $>7, P=0.031$, Stage I + II vs. Stage III + IV $P=0.148$, Fig. 5A). Moreover, with the help of Fisher's extract test, we investigated the different distribution of SRD5A2 expression (strong positive, weak positive, or negative) in different clinicopathological subgroups. We revealed the negative association of SRD5A2 and tumor progression, reflecting by Gleason score $(P=0.013)$, and pathological tumor stage ( $P$ $=0.047$ ) (Table 2). As to ITGA11, we observed an elevated protein expression in advanced stage than early-stage (Gleason < = 7 vs. Gleason > 7, $P=0.067$, Stage I + II vs. Stage III + IV, $P=0.014$, Fig. 5B). Fisher's extract test of the categorical variables illustrated similar results. These patients whose Gleason score higher than 7 or were in tumor stage III and IV showed strong positive staining of ITGA11 (Gleason score, $P=0.049$, Pathological tumor stage, $P=0.022$, Table 3 ). All these results indicated that SRD5A2 protein is negatively associated with the progression of $\mathrm{PCa}$, while ITGA11 protein is positively associated with the advanced stage. 
Table 2

Association between SRD5A2 protein level and pathological features in tissue array.

\begin{tabular}{|c|c|c|c|c|}
\hline \multirow[t]{2}{*}{ Parameter } & \multicolumn{4}{|c|}{ IHC results for SRD5A2 } \\
\hline & $\begin{array}{l}\text { Strong Positive (n, } \\
\%)\end{array}$ & $\begin{array}{l}\text { Weak Positive (n, } \\
\%)\end{array}$ & Negative $(n, \%)$ & $P$ value \\
\hline Age & & & & 0.357 \\
\hline$<60$ & $4(66.67 \%)$ & $1(16.67 \%)$ & $1(16.67 \%)$ & \\
\hline$\geq 60$ & $22(61.11 \%)$ & $12(33.33 \%)$ & $2(5.56 \%)$ & \\
\hline Envelope invasion & & & & 0.304 \\
\hline No & $24(64.86 \%)$ & $11(29.73 \%)$ & $2(5.41 \%)$ & \\
\hline Yes & $2(40.00 \%)$ & $2(40.00 \%)$ & $1(20.00 \%)$ & \\
\hline $\begin{array}{l}\text { Seminal vesicle } \\
\text { invasion }\end{array}$ & & & & 0.395 \\
\hline No & $26(63.41 \%)$ & $12(29.27 \%)$ & $3(7.32 \%)$ & \\
\hline Yes & $0(0.00 \%)$ & $1(100.00 \%)$ & $0(0.00 \%)$ & \\
\hline Gleason score & & & & $0.013^{*}$ \\
\hline$\leq 7$ & $25(67.57 \%)$ & $11(29.73 \%)$ & $1(2.70 \%)$ & \\
\hline$>7$ & $1(20.00 \%)$ & $2(40.00 \%)$ & $2(40.00 \%)$ & \\
\hline Pathology Stage & & & & $0.047 *$ \\
\hline HII & $24(66.67 \%)$ & $11(30.56 \%)$ & $1(2.78 \%)$ & \\
\hline III-IV & $2(33.33 \%)$ & $2(33.33 \%)$ & $2(33.33 \%)$ & \\
\hline$\star, P<0.05$ & & & & \\
\hline
\end{tabular}


Table 3

Association between ITGA11 protein level and pathological features in tissue microarray.

\begin{tabular}{|c|c|c|c|c|}
\hline \multirow[t]{2}{*}{ Parameter } & \multicolumn{4}{|c|}{ IHC results for ITGA11 } \\
\hline & $\begin{array}{l}\text { Strong Positive (n, } \\
\%)\end{array}$ & $\begin{array}{l}\text { Weak Positive (n, } \\
\% \text { ) }\end{array}$ & Negative (n, \%) & $P$ value \\
\hline Age & & & & 0.461 \\
\hline$<60$ & $2(33.33 \%)$ & $3(50.00 \%)$ & $1(16.67 \%)$ & \\
\hline$\geq 60$ & $20(55.56 \%)$ & $12(33.33 \%)$ & $4(11.11 \%)$ & \\
\hline Envelope invasion & & & & 0.667 \\
\hline No & $19(51.35 \%)$ & $14(37.84 \%)$ & $4(10.81 \%)$ & \\
\hline Yes & $3(60.00 \%)$ & $1(20.00 \%)$ & $1(20.00 \%)$ & \\
\hline $\begin{array}{l}\text { Seminal vesicle } \\
\text { invasion }\end{array}$ & & & & 1.000 \\
\hline No & $21(51.22 \%)$ & $15(36.59 \%)$ & $5(12.20 \%)$ & \\
\hline Yes & $1(100.00 \%)$ & $0(0.00 \%)$ & $0(0.00 \%)$ & \\
\hline Gleason score & & & & $0.049 *$ \\
\hline$\leq 7$ & $17(45.95 \%)$ & $20(54.05 \%)$ & & \\
\hline$>7$ & $5(100.00 \%)$ & $0(0.00 \%)$ & & \\
\hline Pathology Stage & & & & $0.022^{*}$ \\
\hline $\mid-H$ & $16(44.44 \%)$ & $20(55.56 \%)$ & & \\
\hline III-IV & $6(100.00 \%)$ & $0(0.00 \%)$ & & \\
\hline
\end{tabular}

\section{5 | Knockdown SRD5A2 and ITGA11 impact prostate cancer cell behaviors}

After knocking down the expression of SRD5A2 (Figure S3), we found the cell proliferation was significantly increased determined by MTT assay and colony formation assays in C4-2 and PC-3 cells (all $P<0.05$, Fig. 6A-B). Since the functional role of SRD5A2 in regulating PCa cell migration and invasion has been investigated by Suruchi Aggarwal et al. ${ }^{22}$, we only focused on the proliferation effects here. On the contrary, we found that silencing ITGA11 expression decreased the cell migration and invasion in C4-2 and PC-3 cells, instead of cell proliferation (all $P<0.05$, Fig. 6C-D, and Fig. 7D). These results confirm the tumor-suppressive role and oncogenic role of SRD5A2 and ITGA11, respectively. 


\section{6 | Exploring the underlying mechanisms of how ITGA11 regulating PCa progression}

Many studies have already illustrated the mechanisms of how the selected 11 genes influence the progression of PCa, while few studies have focused on the role of ITGA11. We conducted Pearson correlation analyses to find out the highly co-expressed genes in each cohort and overlapped these genes derived from all the five cohorts. Then, KEGG analysis was used to enrich the significant signaling pathways. We found that ITGA11 might be involved in the activity regulation of calcium signaling, Rap1 signaling, Ras signaling, and PI3K/Akt signaling pathways (Fig. 7A). Moreover, we collected two gene sets which could reflect the activation status of PI3K/AKT signaling and calculated the NES score of each patient by sSGSEA. We observed that the expression of ITGA11 is positively correlated with the NES score of HALLMARK PI3K/AKT/mTOR signaling gene set $(P<0.05, r=0.43$, Fig. 7B), and REACTOME PI3K/AKT activation gene set $(P<0.05, r=0.34$, Fig. $7 C)$. Besides, we validated the impaction of ITGA11 expression on the activation of PI3K/AKT signaling in vitro. After knocking down the expression of ITGA11, we found that the Ser473 phosphorylated-AKT1/2/3 was significantly decreased instead of total AKT1/2/3, indicating that ITGA11 promotes the migration and invasion of PCa potentially by activating PI3K/AKT signaling (Fig. 7D).

\section{4 | Discussion}

The interpatient heterogeneity in PCa is well recognized ${ }^{23-25}$. However, the molecular stratification of PCa based on predicting biomarkers to guide treatment selection has not yet been applying in the clinic. In our study, we analyzed five datasets derived from GEO (four) and TCGA databases. We firstly employed univariate Cox regression analyses and identified 39 candidate genes that are closely related to the RFS of PCa patients in all the five datasets. The RFS predicting classifier was established by the LASSO Cox regression analysis based MSKCC dataset. The classifier showed satisfying molecular subtyping accuracy determined by the log-rank, K-M, and ROC analyses in both the training and four external validation cohorts. Further, the multivariate analyses suggested that the classifier served as an independent indicator of RFS in a set of cohorts. Notably, the Combined ROC, which synthesized the classifier with clinicopathological features, added prognostic values to the currently available staging system. As studies indicated that highly co-expressed genes potentially have similar biological functions $16-18$, therefore, we conducted Pearson correlation analyses to find out the highly co-expressed genes in each cohort and overlapped these genes derived from all the five cohorts, then employed the KEGG pathway analyses to reveal the underlying mechanisms of these critical candidates influencing tumor progression. For the eleven candidates $22,26-38$, few studies focused on the role of ITGA11 on PCa progression. Thus, we predicted the biological function of ITGA11 through a bioinformatic way indicated above, and the results indicated that ITGA11 might be involved in the activity regulation of calcium signaling, Rap1 signaling, Ras signaling, and PI3K-Akt signaling pathways. Consistently, studies also reported that SRD5A2 regulates cell migration and invasion by indirectly regulating ERK/MAPK pathway ${ }^{22}$. We further investigated the functional role of these two candidates in regulating cancer cell 
fates, as well as the protein expression in clinical samples. Our results confirm the tumor-suppressive role of SRD5A2 and the oncogenic role of ITGA11 in PCa. Nextly, we validated the pathway enrichment results based on co-expressed genes by western blot assay. Our results suggested that silencing ITGA11 suppresses the activity of AKT signaling, indicating that ITGA11 promotes PCa cell progression potentially through activating AKT signaling. Overall, we successfully establish a solid prognosis predicting system.

In recent days, several prognostic classifiers were developed to predict the outcome of PCa patients, based on the clinical features, gene genetics, or epigenetics. Zhao et al. ${ }^{39}$ reported that the PD-L2 is a prognostic biomarker for PCa based on patients, and they also reported that the infiltration of T cells and Macrophages are increased in the poor outcome group, which is also consistent with our work that M2 macrophages link with the unfavorable prognosis, while the immunocytes and clinical feature-based Combined could distinguish the different ending of recurrence $(A U C=0.91){ }^{40}$. Bhargava et al. ${ }^{41}$ illustrated an African-American specifically automated stromal classifier, which has the potential to substantially improve the accuracy of prognosis and risk stratification. Yang et al. ${ }^{42}$ established a 28hypoxia-related-gene prognostic classifier for localized PCa, which could predict the biochemical recurrence and metastasis events. Gleason score $4+3$ is associated with a 3 -fold increased risk of incidence of metastasis at diagnosis than Gleason score $3+4$ though the overall incidence is low 43,44 . In our study, we investigated the value of this classifier in distinguishing patients with Gleason score $3+4$ and $4+3$ subgroups in MSKCC, TCGA-PRAD, GSE70768, and GSE70769 datasets. We observed that patients with Gleason score $4+3$ have a higher risk score as compare with Gleason score $3+4$ patients. Besides, the risk score distinguished the $3+4 / 4+3$ subgroups with good accuracy, a result consistent with Fisher's Extract test. We found that more patients with the high-risk belonged to the Gleason score 4 +3 subgroup. Another limitation of these studies was that their findings were not validated in two more independent cohorts, and the potential mechanisms of how these markers influence tumor progression were not predicted or investigated. Herein, we established an eleven-consensus-gene-based classifier and validated its usage in five independent cohorts. We also predicted the potential mechanisms through bioinformatic ways. Thus, our findings are stable and convincing.

The advantages of the current study are summarized and presented as follows. Firstly, we identified a 39consensus-gene-cluster from five independent cohorts, and with the help of the LASSO Cox regression analysis, we chose the most suitable eleven candidates to establish the RFS predicting classifier. The classifier showed satisfying molecular prognostic subtyping accuracy determined by the log-rank, K-M, and ROC analyses in all five cohorts. Second, we proved that the classifier serves as an independent indicator of RFS in a set of cohorts and adds prognostic value to the currently available staging system. Thirdly, we predicted the potential mechanisms of how these critical candidates influence the progression of PCa, which would benefit the development of targeted drugs. However, lack of survival analysis in our samples and lack of systematic functional studies to prove the function and mechanisms of these consensus genes are the major limitations of the current study. 
Our study successfully subtypes PCa patients with different prognostic outcomes based on an elevenconsensus-gene-based classifier containing 1,046 cases. The classifier adds prognostic value to the currently available staging system. These findings propel the development of personalized medicine for PCa patients shortly.

\section{Abbreviations}

PCa, prostate cancer; CRPC, castration-resistant PCa; RFS, recurrence-free survival; ROC, receiver operating characteristic; IHC, immunohistochemistry; ssGSEA, single sample gene set enrichment analysis; NES, normalized enrichment score; LASSO, least absolute shrinkage and selection operator.

\section{Declarations}

\section{Competing interests}

The authors declare that there is no conflict of interest.

\section{Data availability}

All data used in this work can be acquired from the Gene-Expression Omnibus (GEO; https://www.ncbi.nlm.nih.gov/geo/) and the GDC portal (https://portal.gdc.cancer.gov/).

\section{References}

1. Siegel, R. L., Miller, K. D. \& Jemal, A. Cancer statistics, 2019. CA Cancer J Clin 69, 7-34, doi:10.3322/caac.21551 (2019).

2. Rawla, P. Epidemiology of Prostate Cancer. World J Oncol 10, 63-89, doi:10.14740/wjon1191 (2019).

3. Shao, N. et al. Immunotherapy and endothelin receptor antagonists for treatment of castrationresistant prostate cancer. Int J Cancer 133, 1743-1750, doi:10.1002/ijc.28162 (2013).

4. Mansinho, A., Macedo, D., Fernandes, I. \& Costa, L. Castration-Resistant Prostate Cancer: Mechanisms, Targets and Treatment. Adv Exp Med Biol 1096, 117-133, doi:10.1007/978-3-31999286-0_7 (2018).

5. Jang, W. S. et al. The prognostic impact of downgrading and upgrading from biopsy to radical prostatectomy among men with Gleason score 7 prostate cancer. Prostate 79, 1805-1810, doi:10.1002/pros.23905 (2019).

6. Tilki, D. et al. Surgery vs Radiotherapy in the Management of Biopsy Gleason Score 9-10 Prostate Cancer and the Risk of Mortality. JAMA Oncol 5, 213-220, doi:10.1001/jamaoncol.2018.4836 (2019).

7. Egevad, L., Granfors, T., Karlberg, L., Bergh, A. \& Stattin, P. Percent Gleason grade 4/5 as prognostic factor in prostate cancer diagnosed at transurethral resection. J Urol 168, 509-513 (2002). 
8. Wright, J. L. et al. Prostate cancer specific mortality and Gleason 7 disease differences in prostate cancer outcomes between cases with Gleason $4+3$ and Gleason $3+4$ tumors in a population based cohort. J Uro/ 182, 2702-2707, doi:10.1016/j.juro.2009.08.026 (2009).

9. Fischer, S. et al. Do all men with pathological Gleason score 8-10 prostate cancer have poor outcomes? Results from the SEARCH database. 118, 250-257.

10. Chan, T. Y., Partin, A. W., Walsh, P. C. \& Epstein, J. I. Prognostic significance of Gleason score $3+4$ versus Gleason score $4+3$ tumor at radical prostatectomy. Urology $56,823-827$, doi:10.1016/s0090-4295(00)00753-6 (2000).

11. Dall'Era, M. A. et al. Active surveillance for prostate cancer: a systematic review of the literature. Eur Uro/ 62, 976-983, doi:10.1016/j.eururo.2012.05.072 (2012).

12. Kwa, M., Makris, A. \& Esteva, F. J. Clinical utility of gene-expression signatures in early stage breast cancer. Nat Rev Clin Oncol 14, 595-610, doi:10.1038/nrclinonc.2017.74 (2017).

13. Yin, Y. et al. N-Myc promotes therapeutic resistance development of neuroendocrine prostate cancer by differentially regulating miR-421/ATM pathway. Mol Cancer 18, 11, doi:10.1186/s12943-0190941-2 (2019).

14. Chen, J. et al. The Hypermethylation of Foxp3 Promoter Impairs the Function of Treg Cells in EAP. Inflammation 42, 1705-1718, doi:10.1007/s10753-019-01030-0 (2019).

15. Yi, B. et al. Overexpression of RhoGDI2 correlates with the progression and prognosis of pancreatic carcinoma. Oncol Rep 33, 1201-1206, doi:10.3892/or.2015.3707 (2015).

16. Wang, J. et al. Single-Cell Co-expression Analysis Reveals Distinct Functional Modules, Co-regulation Mechanisms and Clinical Outcomes. PLoS Comput Biol 12, e1004892-e1004892, doi:10.1371/journal.pcbi.1004892 (2016).

17. Niemira, M. et al. Molecular Signature of Subtypes of Non-Small-Cell Lung Cancer by Large-Scale Transcriptional Profiling: Identification of Key Modules and Genes by Weighted Gene Co-Expression Network Analysis (WGCNA). Cancers 12, 37, doi:10.3390/cancers12010037 (2019).

18. Di, Y., Chen, D., Yu, W. \& Yan, L. Bladder cancer stage-associated hub genes revealed by WGCNA coexpression network analysis. Hereditas 156, 7-7, doi:10.1186/s41065-019-0083-y (2019).

19. Yu, G., Wang, L. G., Han, Y. \& He, Q. Y. clusterProfiler: an R package for comparing biological themes among gene clusters. Omics: a journal of integrative biology 16, 284-287, doi:10.1089/omi.2011.0118 (2012).

20. Hanzelmann, S., Castelo, R. \& Guinney, J. GSVA: gene set variation analysis for microarray and RNAseq data. BMC Bioinformatics 14, 7, doi:10.1186/1471-2105-14-7 (2013).

21. Barbie, D. A. et al. Systematic RNA interference reveals that oncogenic KRAS-driven cancers require TBK1. Nature 462, 108-112, doi:10.1038/nature08460 (2009).

22. Aggarwal, S., Singh, M., Kumar, A. \& Mukhopadhyay, T. SRD5A2 gene expression inhibits cell migration and invasion in prostate cancer cell line via F-actin reorganization. Mol Cell Biochem 408, 15-23, doi:10.1007/s11010-015-2478-z (2015). 
23. Rahmani, E. et al. Sparse PCA corrects for cell type heterogeneity in epigenome-wide association studies. Nat Methods 13, 443-445, doi:10.1038/nmeth.3809 (2016).

24. Finak, G. et al. MAST: a flexible statistical framework for assessing transcriptional changes and characterizing heterogeneity in single-cell RNA sequencing data. Genome Bio/ 16, 278, doi:10.1186/s13059-015-0844-5 (2015).

25. Armenia, J. et al. The long tail of oncogenic drivers in prostate cancer. Nat Genet 50, 645-651, doi:10.1038/s41588-018-0078-z (2018).

26. Hu, H. et al. RANKL expression in normal and malignant breast tissue responds to progesterone and is up-regulated during the luteal phase. Breast cancer research and treatment 146, 515-523, doi:10.1007/s10549-014-3049-9 (2014).

27. Puyo, S. et al. Gene expression signature predicting high-grade prostate cancer responses to oxaliplatin. Mol Pharmacol 82, 1205-1216, doi:10.1124/mol.112.080333 (2012).

28. Pudova, E. A. et al. Differentially Expressed Genes Associated With Prognosis in Locally Advanced Lymph Node-Negative Prostate Cancer. Front Genet 10, 730, doi:10.3389/fgene.2019.00730 (2019).

29. Gonzalez-Gronow, M., Grenett, H. E., Gawdi, G. \& Pizzo, S. V. Angiostatin directly inhibits human prostate tumor cell invasion by blocking plasminogen binding to its cellular receptor, CD26. Exp Cell Res 303, 22-31, doi:10.1016/j.yexcr.2004.09.008 (2005).

30. Russo, J. W. et al. Downregulation of Dipeptidyl Peptidase 4 Accelerates Progression to CastrationResistant Prostate Cancer. Cancer Res 78, 6354-6362, doi:10.1158/0008-5472.CAN-18-0687 (2018).

31. Shah, C. et al. Impact of DPP4 Inhibitors in Survival of Patients With Prostate, Pancreas, and Breast Cancer. Front Oncol 10, 405, doi:10.3389/fonc.2020.00405 (2020).

32. Tiger, C. F., Fougerousse, F., Grundstrom, G., Velling, T. \& Gullberg, D. alpha11beta1 integrin is a receptor for interstitial collagens involved in cell migration and collagen reorganization on mesenchymal nonmuscle cells. Dev Bio/ 237, 116-129, doi:10.1006/dbio.2001.0363 (2001).

33. Sun, T. et al. MiR-221 promotes the development of androgen independence in prostate cancer cells via downregulation of HECTD2 and RAB1A. Oncogene 33, 2790-2800, doi:10.1038/onc.2013.230 (2014).

34. Orr, B. et al. Identification of stromally expressed molecules in the prostate by tag-profiling of cancerassociated fibroblasts, normal fibroblasts and fetal prostate. Oncogene 31, 1130-1142, doi:10.1038/onc.2011.312 (2012).

35. Sampson, N. et al. Inhibition of Nox4-dependent ROS signaling attenuates prostate fibroblast activation and abrogates stromal-mediated protumorigenic interactions. Int J Cancer 143, 383-395, doi:10.1002/ijc.31316 (2018).

36. Penet, M. F. et al. Structure and Function of a Prostate Cancer Dissemination-Permissive Extracellular Matrix. Clin Cancer Res 23, 2245-2254, doi:10.1158/1078-0432.CCR-16-1516 (2017).

37. Sabherwal, Y. et al. PDEF downregulates stathmin expression in prostate cancer. International journal of oncology 40, 1889-1899, doi:10.3892/ijo.2012.1392 (2012). 
38. (!!! INVALID CITATION !!!).

39. Zhao, S. G. et al. The Immune Landscape of Prostate Cancer and Nomination of PD-L2 as a Potential Therapeutic Target. J Nat/ Cancer Inst 111, 301-310, doi:10.1093/jnci/djy141 (2019).

40. Meng, J. et al. The establishment of immune infiltration based novel recurrence predicting nomogram in prostate cancer. Cancer medicine 8, 5202-5213, doi:10.1002/cam4.2433 (2019).

41. Bhargava, H. K. et al. Computationally Derived Image Signature of Stromal Morphology Is Prognostic of Prostate Cancer Recurrence Following Prostatectomy in African American Patients. Clin Cancer Res, doi:10.1158/1078-0432.CCR-19-2659 (2020).

42. Yang, L. et al. Development and Validation of a 28-gene Hypoxia-related Prognostic Signature for Localized Prostate Cancer. EBioMedicine 31, 182-189, doi:10.1016/j.ebiom.2018.04.019 (2018).

43. Kamel, M. H., Khalil, M. I., Alobuia, W. M., Su, J. \& Davis, R. Incidence of metastasis and prostatespecific antigen levels at diagnosis in Gleason $3+4$ versus $4+3$ prostate cancer. Urol Ann 10, 203208, doi:10.4103/UA.UA_124_17 (2018).

44. Zhu, X., Gou, X. \& Zhou, M. Nomograms Predict Survival Advantages of Gleason Score $3+4$ Over $4+$ 3 for Prostate Cancer: A SEER-Based Study. Frontiers in Oncology 9, doi:10.3389/fonc.2019.00646 (2019).

\section{Figures}


a

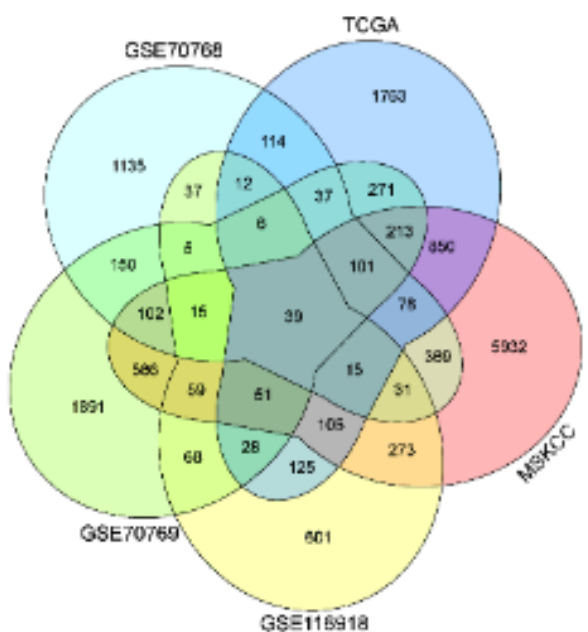

b

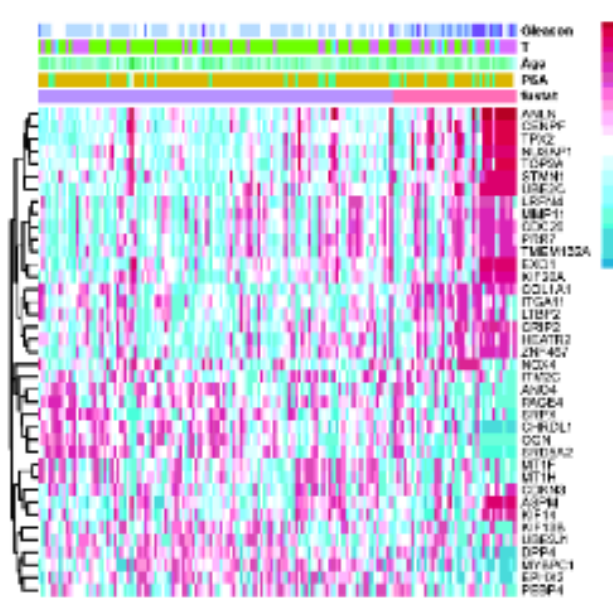

C

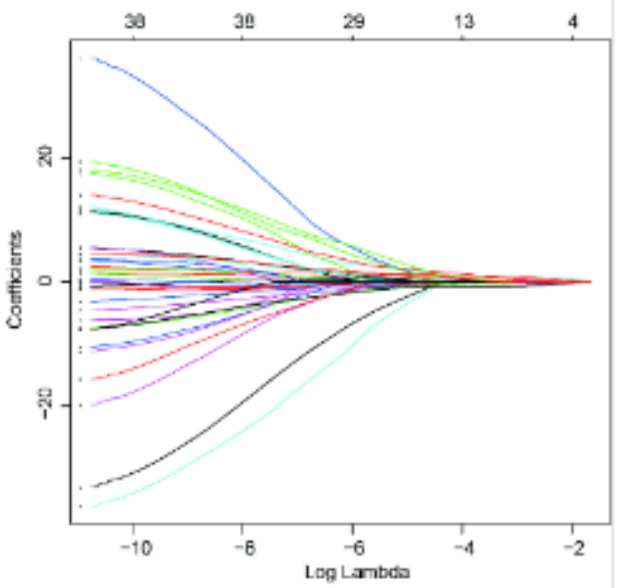

d

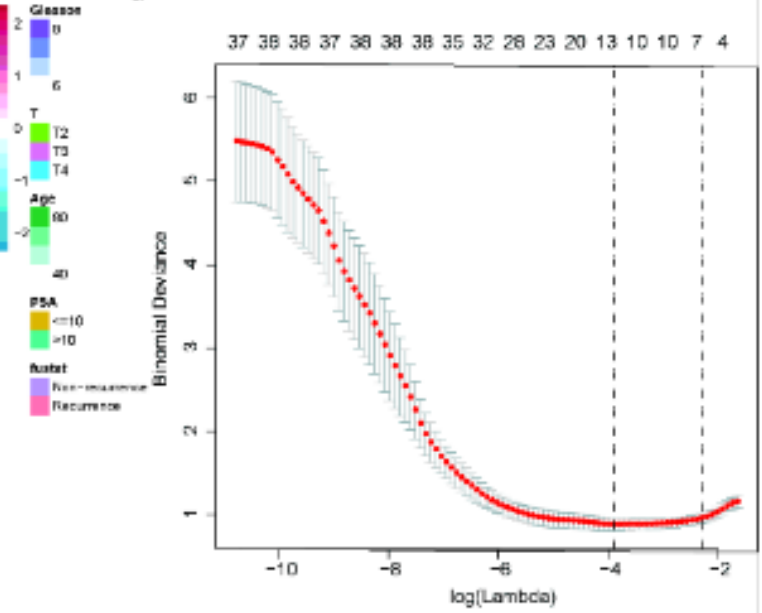

e
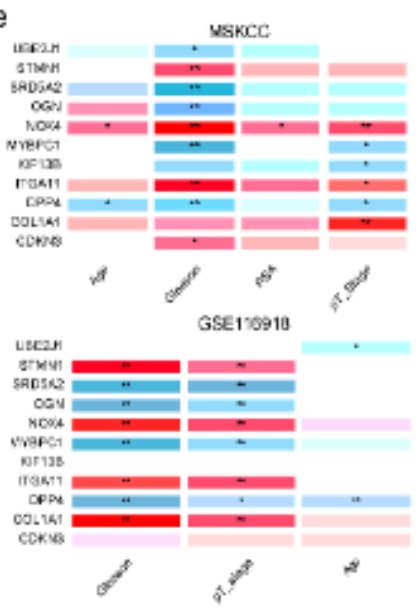

Fig. 1
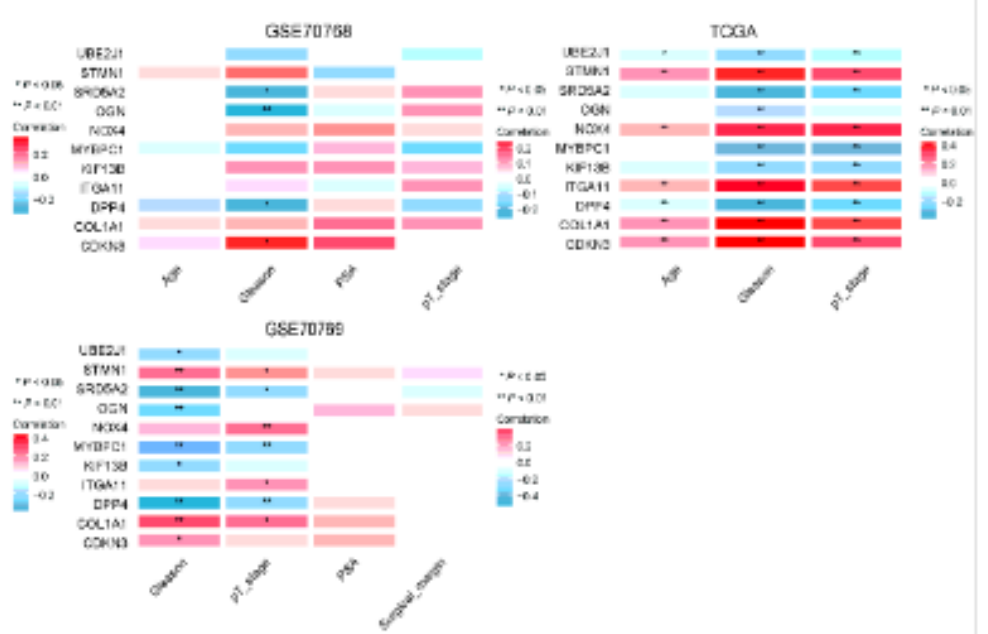

Prtar

$=2$

\section{Figure 1}

Selection of the consensus recurrence-free survival-related genes and establishment of the classifier. (a) Venn diagram showed the overlapping 39 recurrence-free survival (RFS)-associated genes with a P-value $<0.05$ in the five independent datasets. (b) Heatmap showed the expression landscape of the 39 RFSrelated genes in MSKCC dataset, blanks/gaps indicated the missing value. (c) LASSO analysis revealed the coefficients in the model at varying levels of penalization plotted against the log (Lambda) sequence. 
(d) Partial likelihood deviance was plotted versus log(Lambda). The vertical dotted line indicates the lambda value with the minimum error and the largest lambda value where the deviance is within one SE of the minimum. (e) Correlation analyses between the expression of the eleven consensus genes and clinicopathological features in MSKCC, GSE70768, TCGA-PRAD, GSE116918, and GSE70769 cohorts. *, P $<0.05, * \star, P<0.01$

a

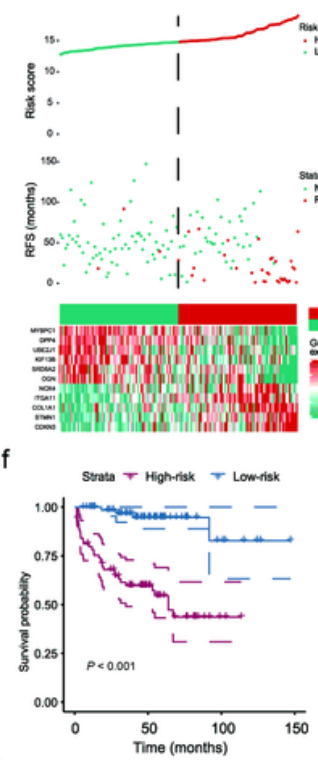

k

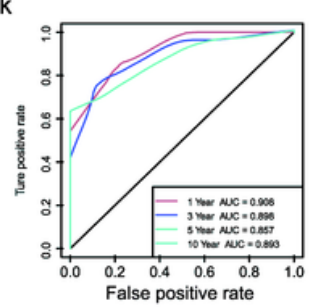

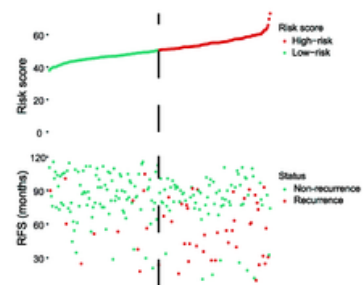

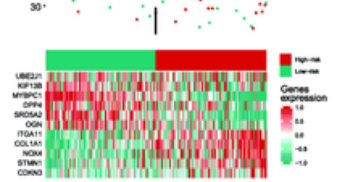

g

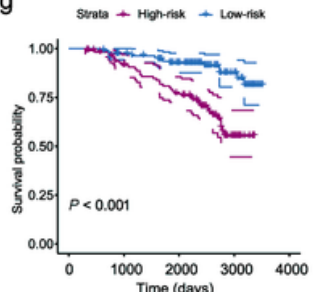

I

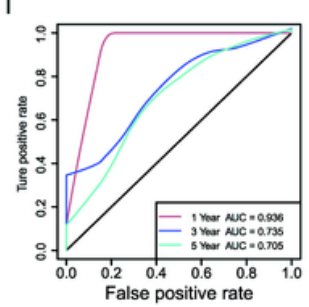

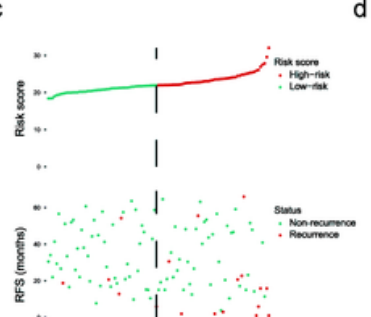

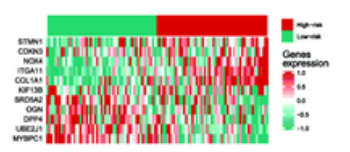

h Strats + High-riss + Low-risk

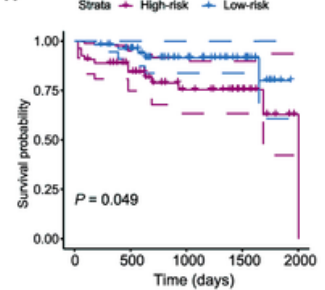

$\mathrm{m}$

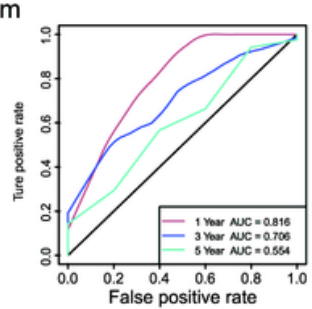

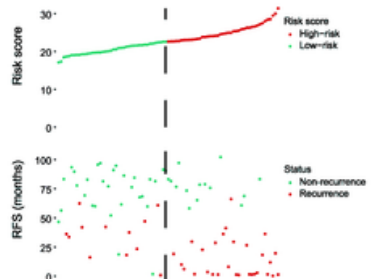
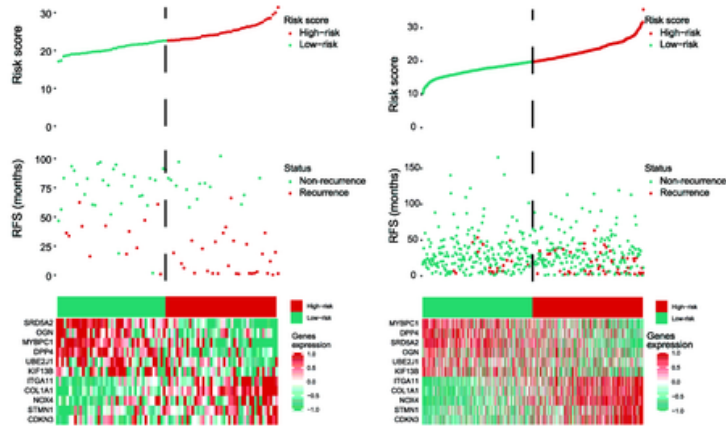

Strata + High-nisk + Low-risk
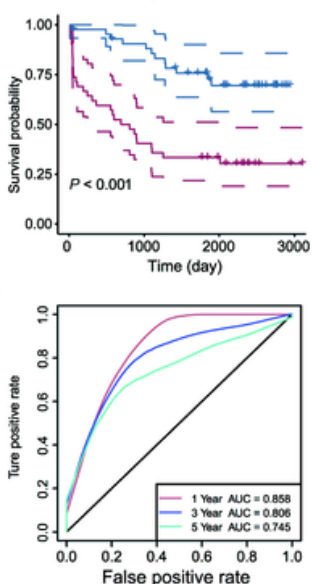

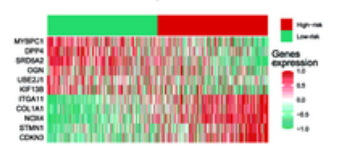

j Stata + High-riak + Low-risk

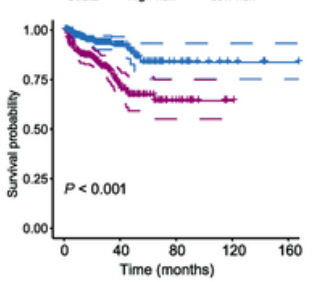

0

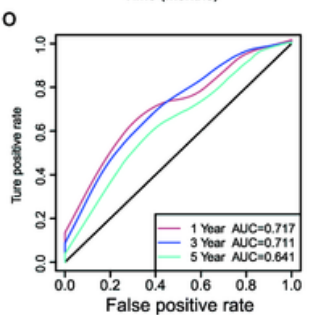

Fig. 2.

\section{Figure 2}

The prognostic value of the eleven-consensus-gene-based classifier. (a-e) The plots displayed the risk classification (upper), corresponding to the distribution of real recurrent samples (middle), and the heatmap displayed the expression of the eleven candidates in different risk subgroups (lower) in MSKCC, GSE116918, GSE70768, GSE70769, and TCGA-PRAD cohorts. (f-j) Kaplan-Meier plots showed the distinguishing value of favorable and poor recurrence-free survival in the MSKCC, GSE116918, GSE70768, GSE70769, and TCGA-PRAD cohorts. (k-o) ROC curves showed the predicting accuracy of the eleven-consensus-gene based classifier for predicting the RFS prognosis of prostate cancer patients in the MSKCC, GSE116918, GSE70768, GSE70769, and TCGA-PRAD cohorts. 


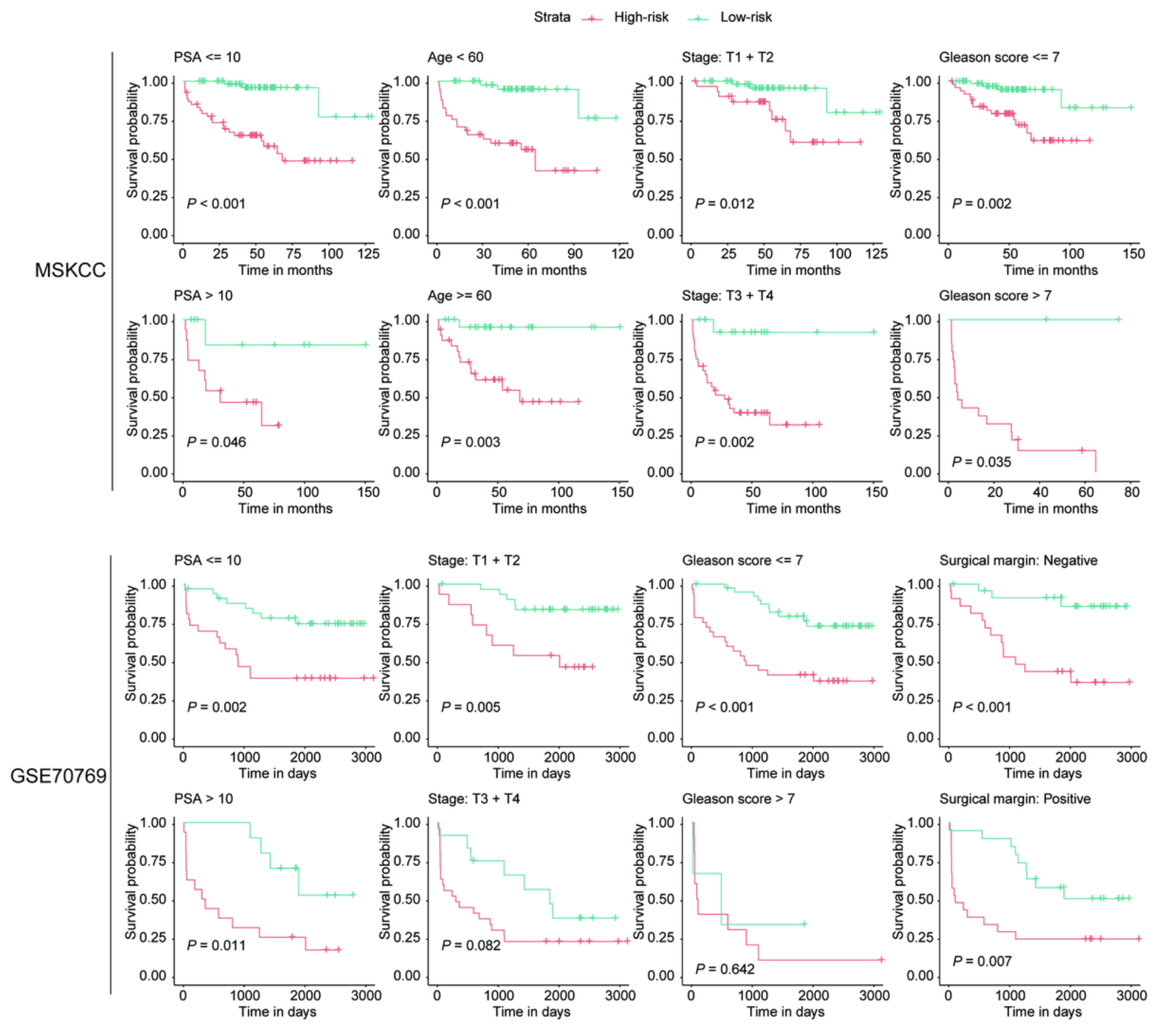

Fig. 3

Figure 3

Kaplan-Meier analyses showed the usage of the eleven-consensus-gene-based classifier in different clinicopathological subgroups in MSKCC and GSE70769 cohorts. Survival probability reflected the recurrence-free survival, $\mathrm{P}$ value obtained from the log-rank test. 
a

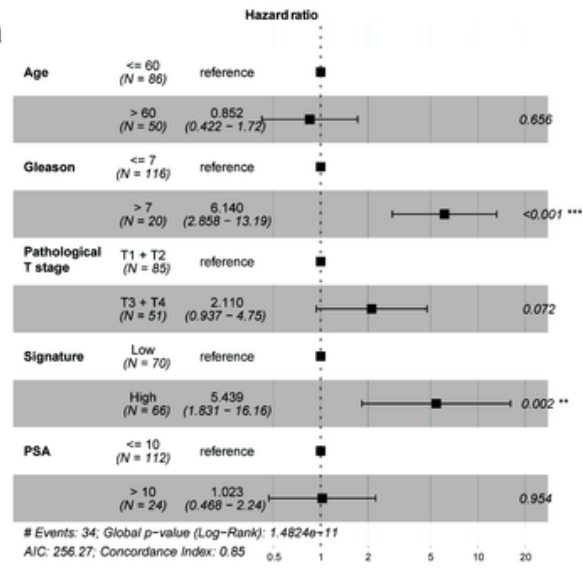

b

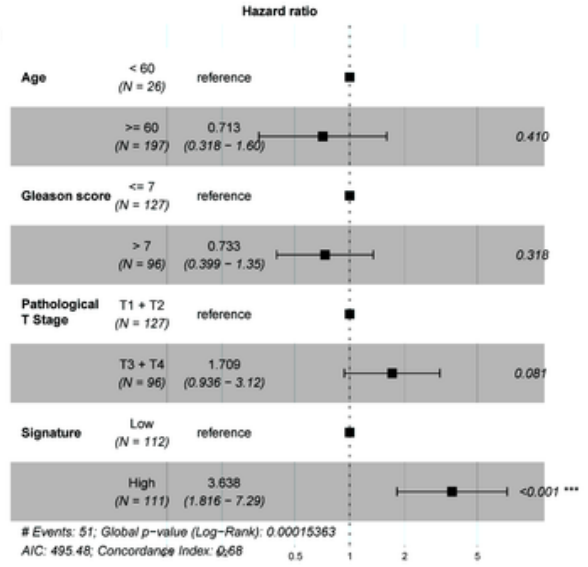

c

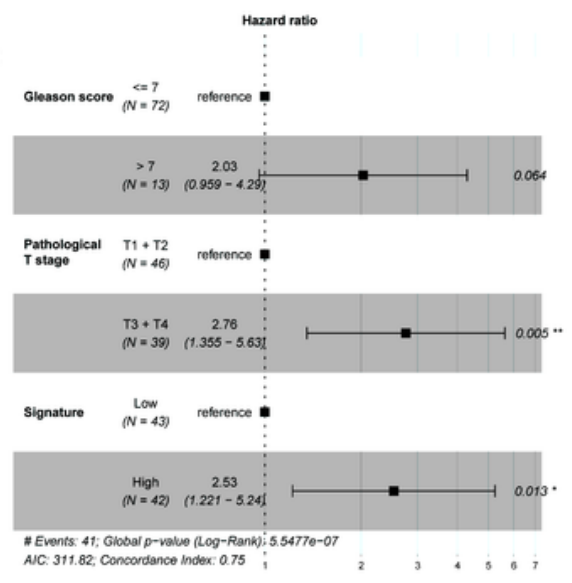

d

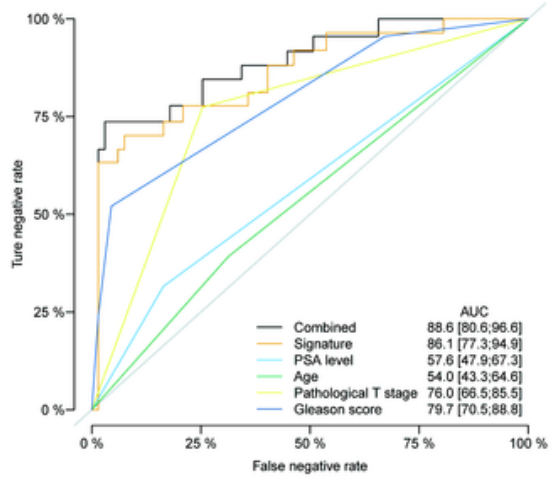

e

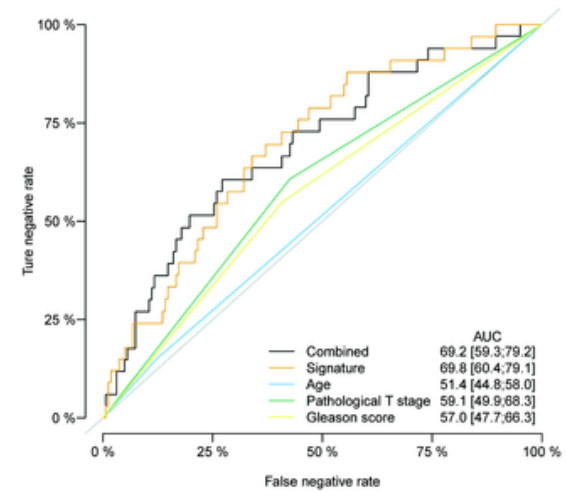

f

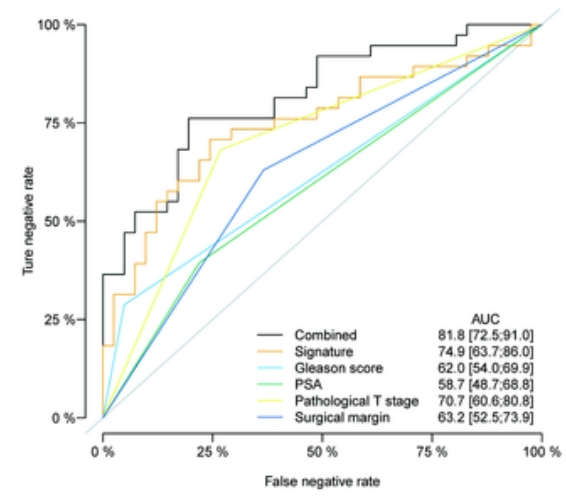

Fig. 4

\section{Figure 4}

Multivariate regression and combined receiver operating characteristic (ROC) curve analyses. We performed multivariate regression analyses and found that the eleven-consensus-gene-based classifier serves as an independent indicator in Memorial Sloan-Kettering Cancer Center (MSKCC) (a), GSE116918 (b), and GSE70769 (c), respectively. Besides, we conducted Combined ROC analyses to compare the predictive values between clinicopathological features and our classifier, as well as to reveal whether a 
synthesized effect existed. We found that the Combined ROC obtained an increased predictive value in the MSKCC (d), GSE116918 (e), GSE70769 (f) cohorts.

a

Gleason score $\leq 7$

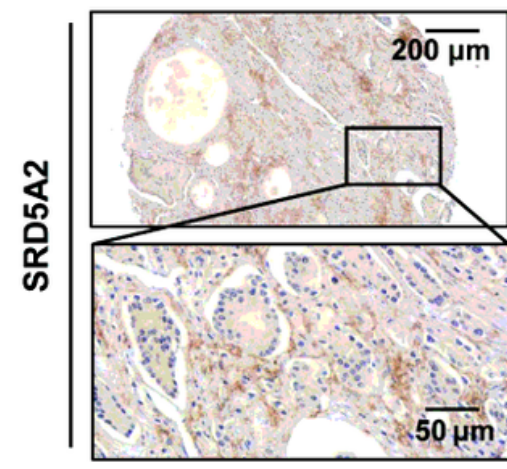

b

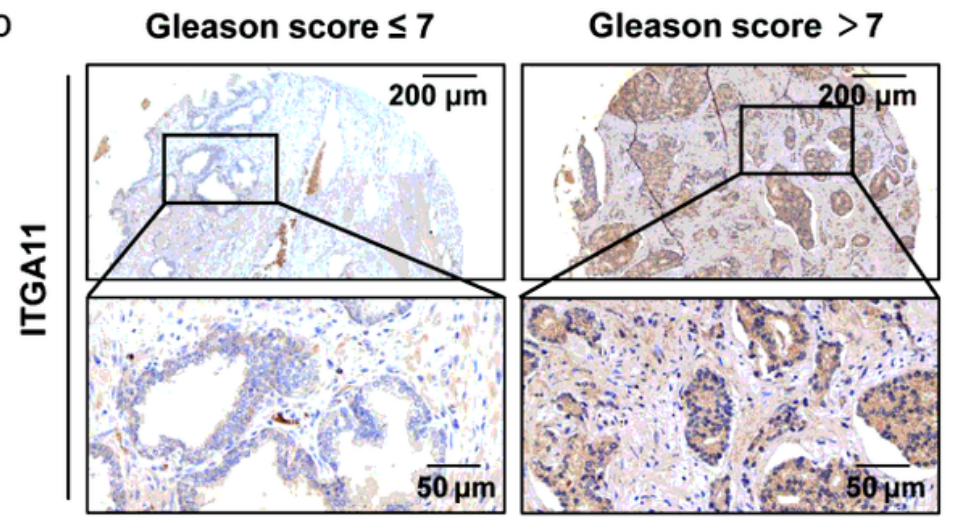

Gleason score $>7$
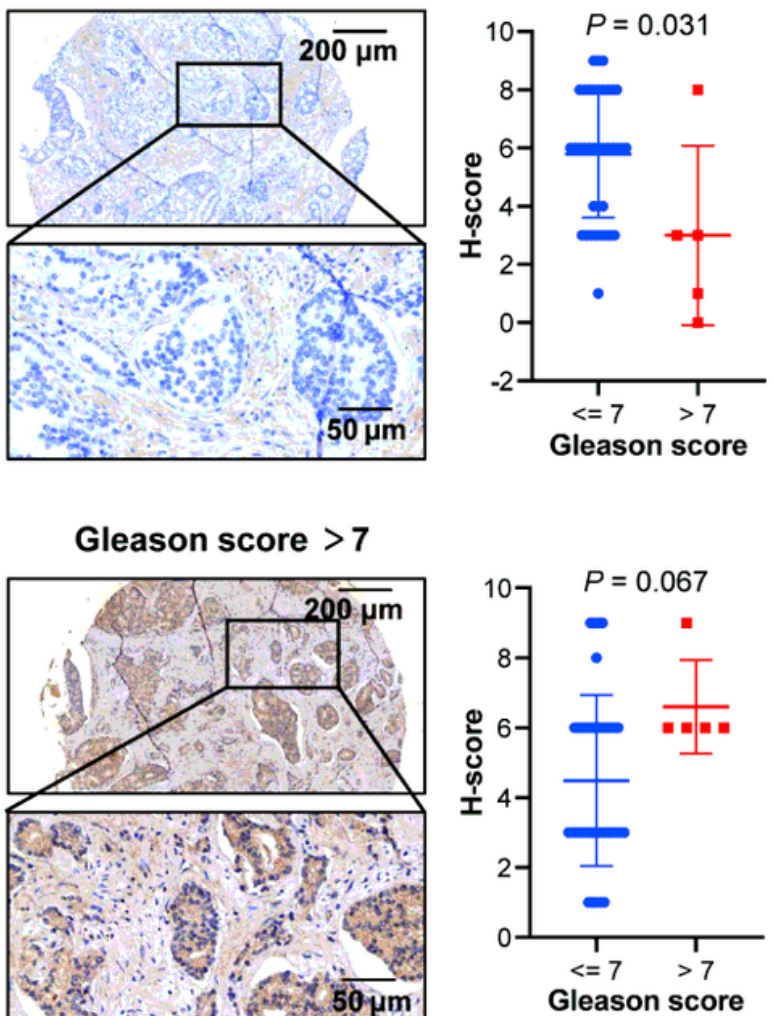
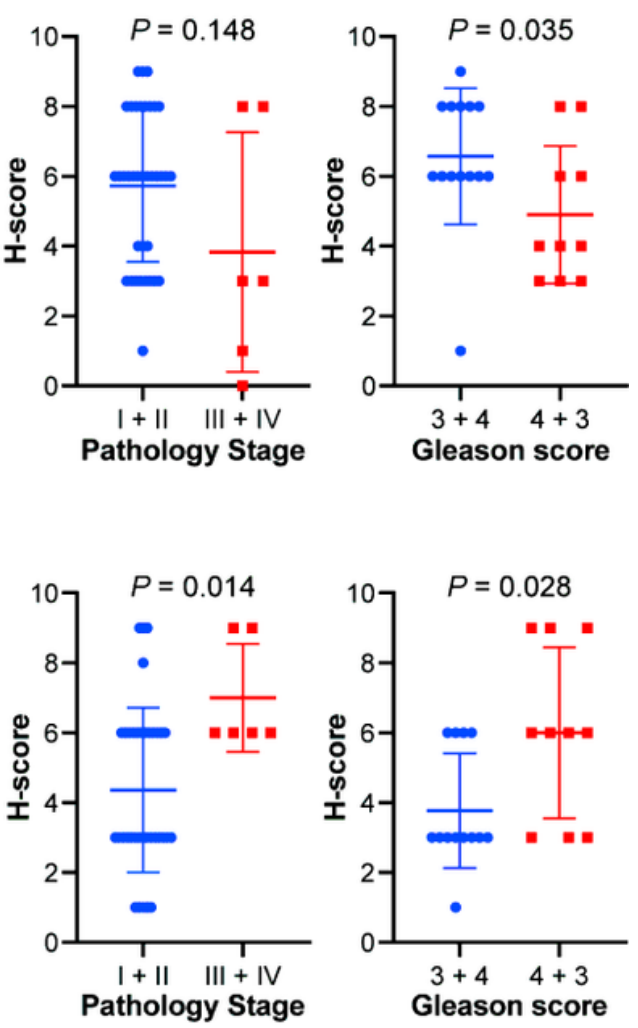

Fig.5

\section{Figure 5}

Immunohistochemistry validation of SRD5A2 and ITGA11. (a) The representative pictures showed the different protein levels of SRD5A2 in prostate cancer patients with different Gleason score. The quantity comparison of H-score also performed in Gleason and pathological tumor stage subgroups. (b) The representative pictures showed the different protein levels of ITGA11 in prostate cancer patients with different Gleason score. The quantity comparison of staining density also performed in Gleason and pathological tumor stage subgroups. 
a
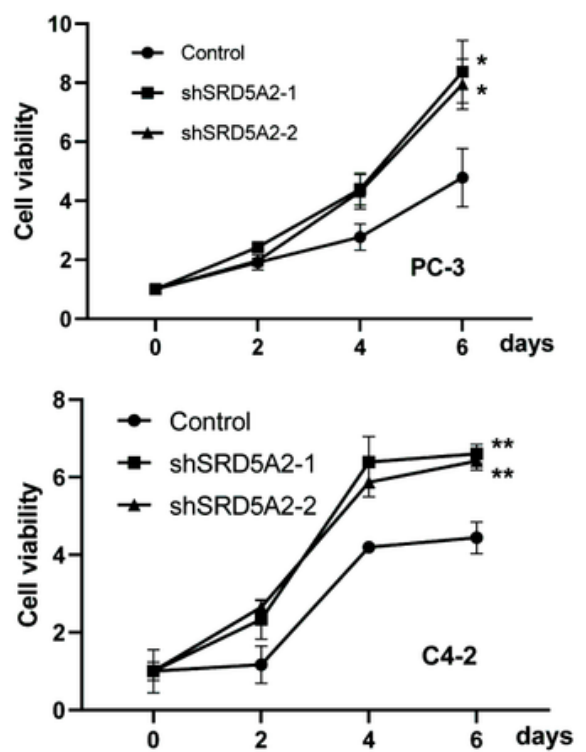

c

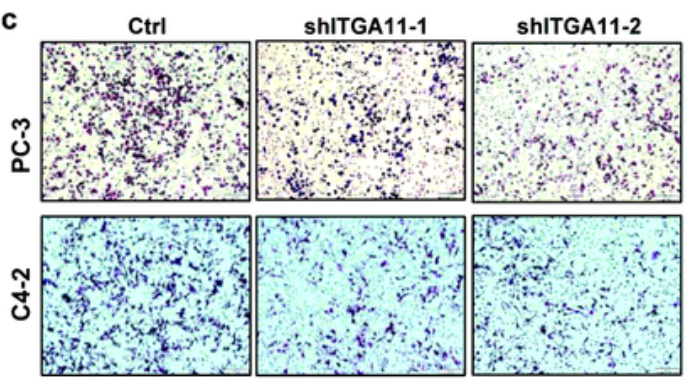

d

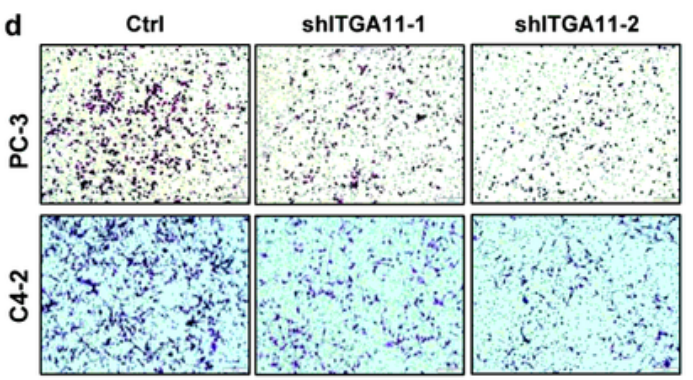

b Ctrl shSRD5A2-1 shSRD5A2-2

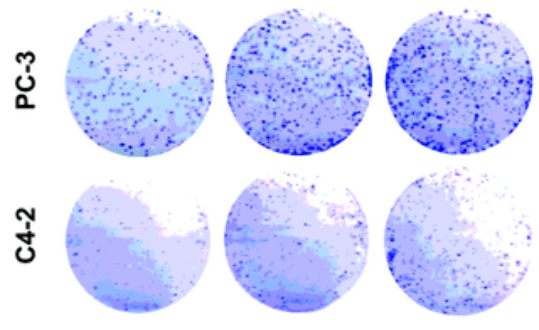

PC-3
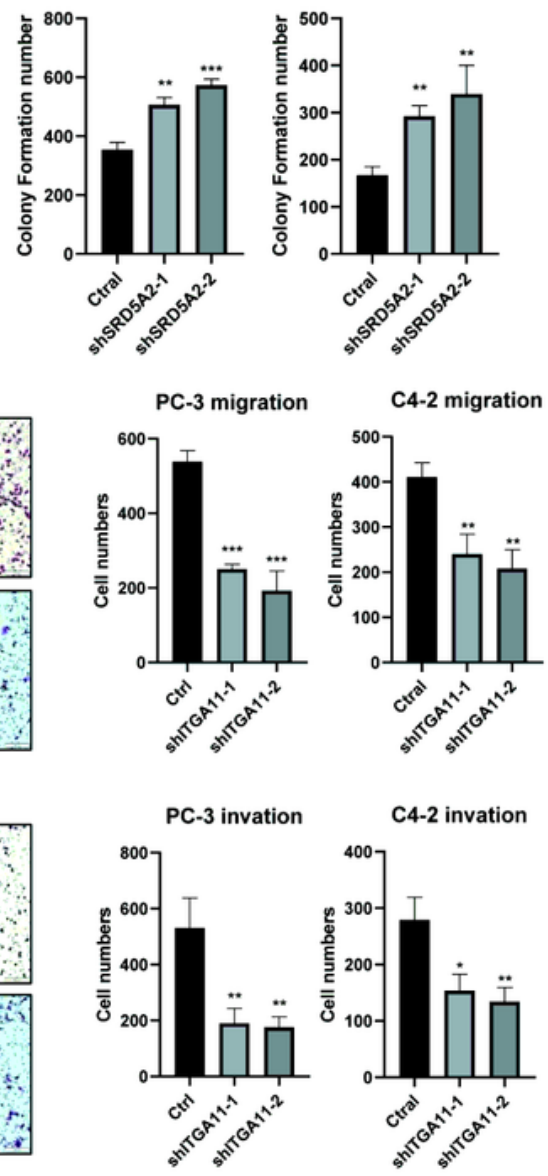

Fig. 6

\section{Figure 6}

Knockdown of SRD5A2 and ITGA11 altering the cell proliferation, migration, and invasion. Knockdown SRD5A2 promoted cell proliferation in both PC-3 and C4-2 cell lines, assessed by MTT assay (a) and colony formation assay (b). Knockdown ITGA11 inhibited the cell migration in PC-3 and C4-2 cell lines (c), as well as the cell invasion (d). ${ }^{*}, \mathrm{P}<0.05, * \star, P<0.01, \star \star \star, ~ P<0.001$. 


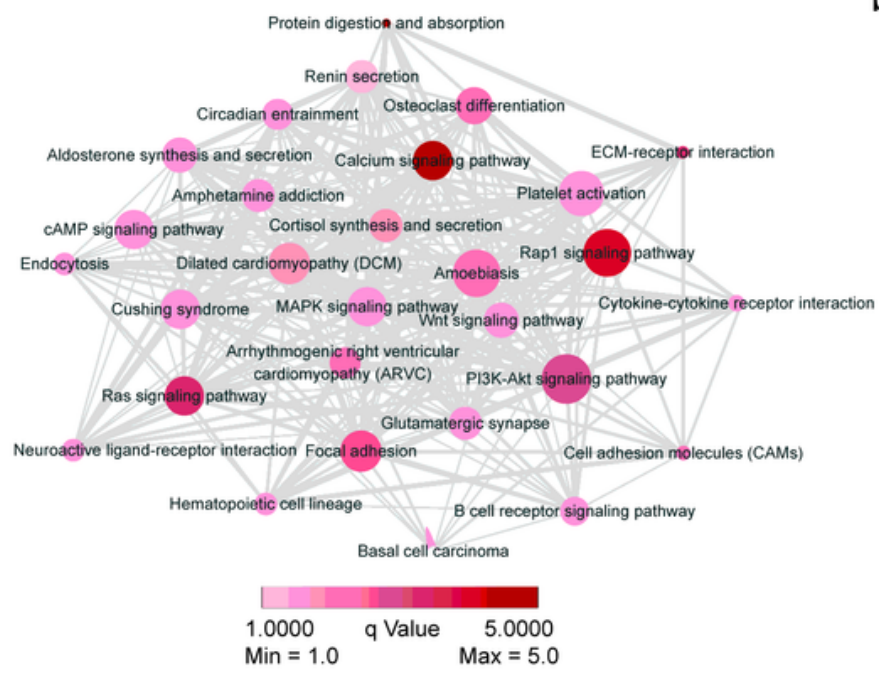

C

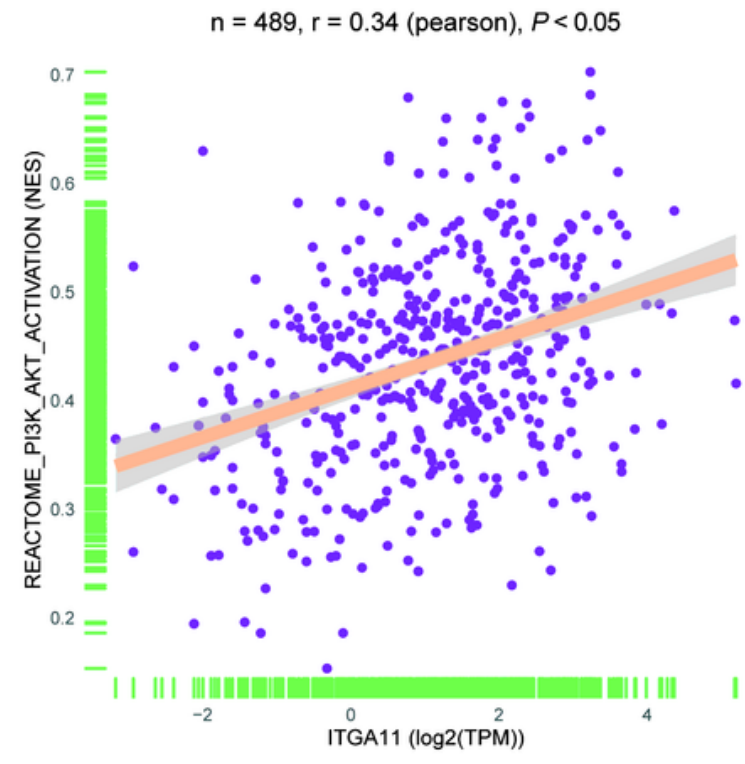

e

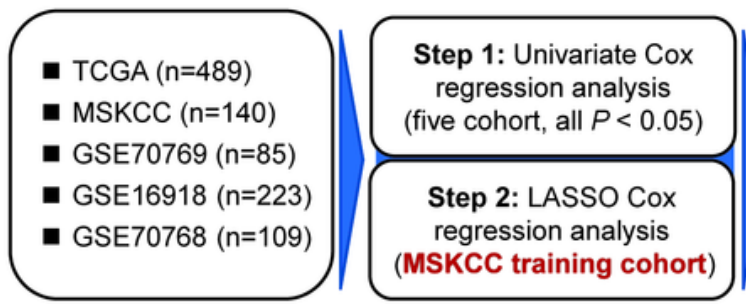

b

$$
n=489, r=0.43 \text { (pearson), } P<0.05
$$

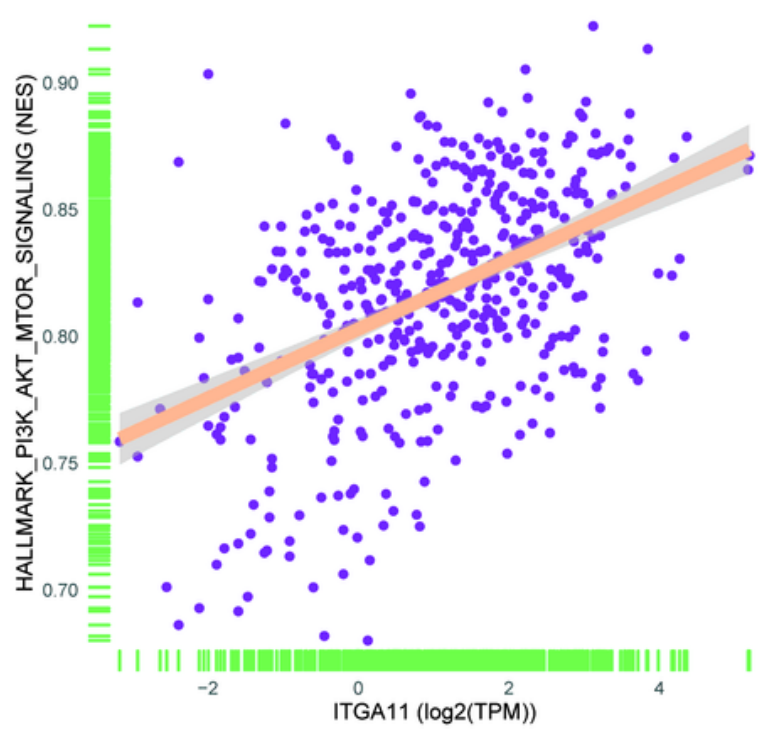

d

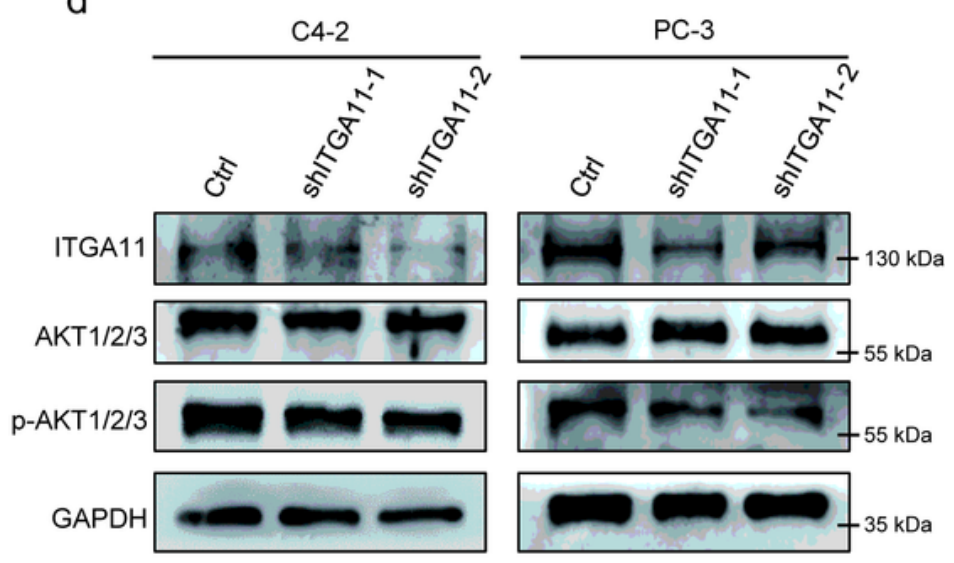

Step 4: Validated in externa cohorts: TCGA, GSE70768, GSE70769, GSE116918

Step 3: Eleven-consensusgene-based signature

\section{Step 5:}

- Mechanism prediction and western blot validation

- Immunohistochemistry validation

- Immunohistochemistry validation
- Biological functional validation

Fig. 7

\section{Figure 7}

ITGA11 inhibits prostate cancer cell migration and invasion through PI3K/AKT signaling. (a) Overlapped co-expressed genes of ITGA11 in five cohorts and KEGG pathway enrichment analysis. (b) Correlation between ITGA11 expression and the activation level of PI3K/AKT signaling assessed by HALLMARK $\mathrm{PI3K} / \mathrm{AKT} / \mathrm{mTOR}$ signaling. (c) Correlation between ITGA11 expression and the activation level of 
PI3K/AKT signaling assessed by REACTOME PI3K/AKT activation. (d) Western blot validated the inhibition of Ser473 p-AKT1/2/3 activation via knockdown the shITGA11 in both C4-2 and PC-3 cells.

\section{Supplementary Files}

This is a list of supplementary files associated with this preprint. Click to download.

- TableS1.doc

- Tables2.xIsx

- Fig.S1.pdf

- Fig.S2.pdf

- Fig.S3.pdf

- Fig.S4.pdf

- SupplementaryFigures.pdf

- reportingsummary.pdf 